\title{
Actinomyces urogenitalis
}

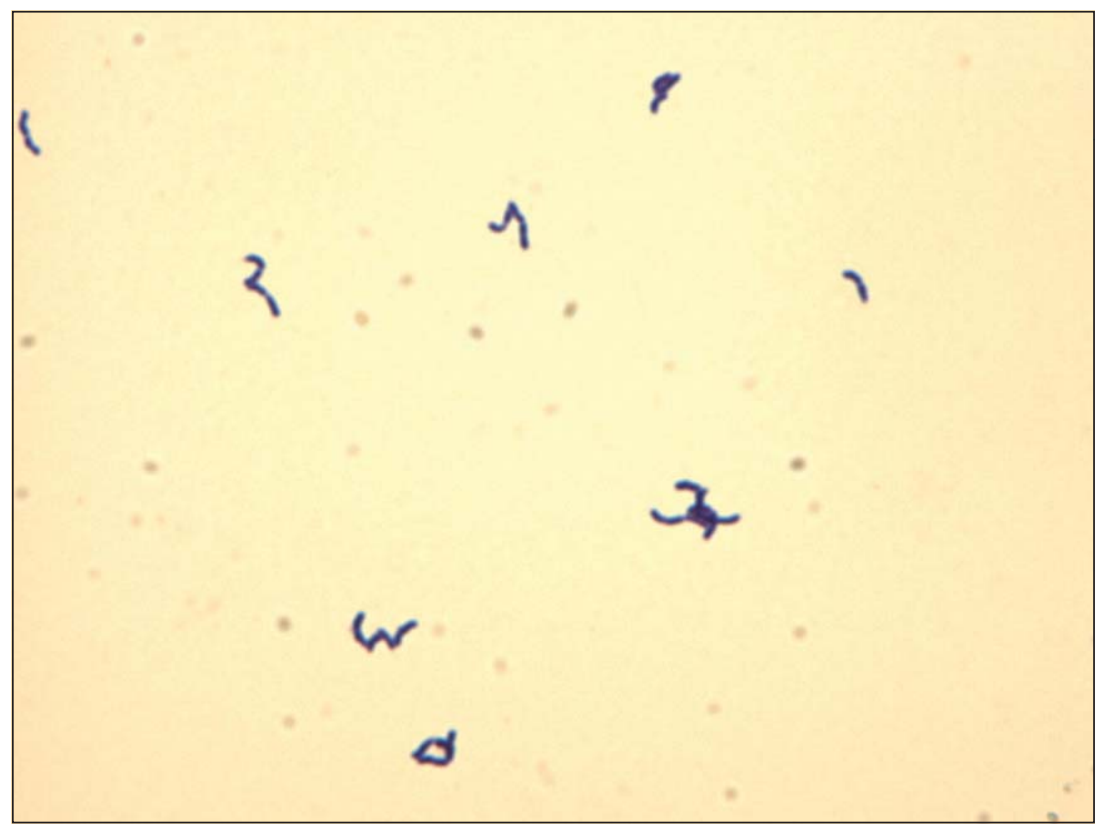

Figura 1.

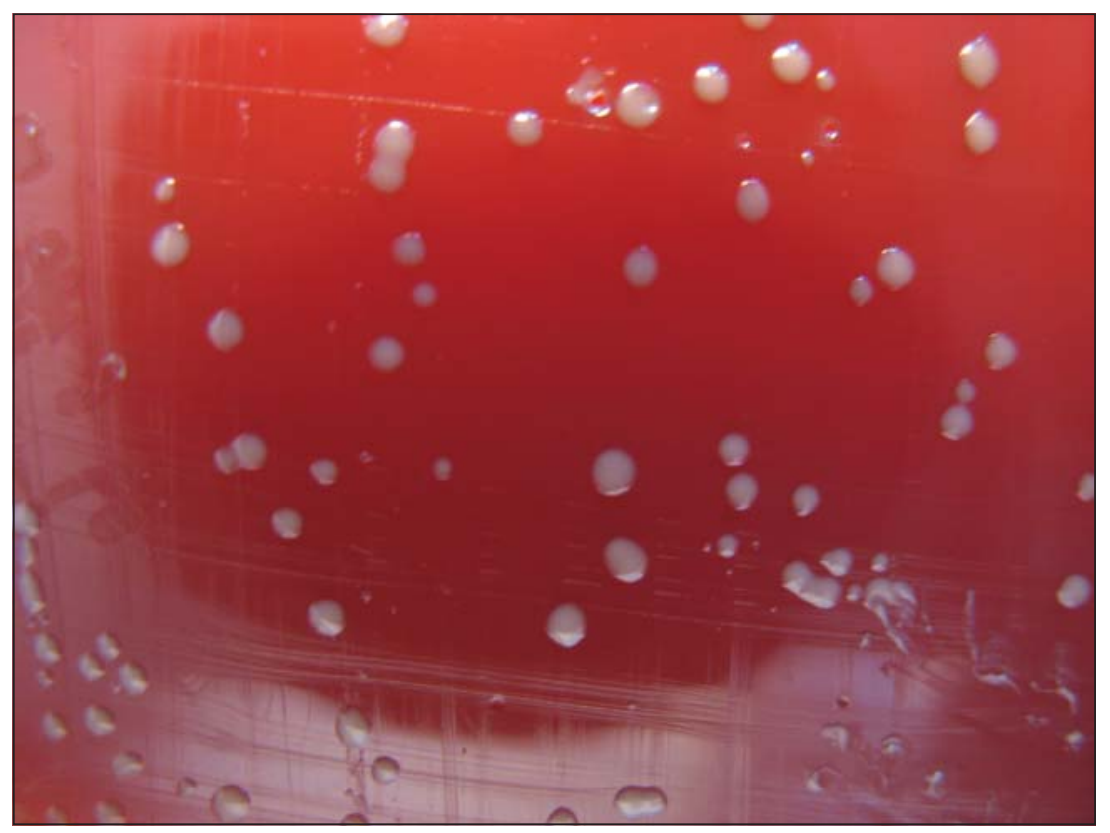

Figura 2. 


\title{
Actinomyces urogenitalis
}

Actinomyces urogenitalis es un bacilo grampositivo recto a ligeramente curvo, con una capacidad rudimentaria de ramificar (Figura 1). Es anaerobio facultativo y catalasa negativa. Se desarrolla en agar sangre originando colonias pequeñas, grises y brillantes desde las 48 horas de incubación en 3\% de $\mathrm{CO}_{2}$. En anaerobiosis forma colonias de 2-3 mm, blanquecino-amarillentas, brillantes, ligeramente convexas y de bordes regulares (Figura 2). Hidroliza la esculina, pero no la gelatina, urea o hipurato. Reduce los nitratos a nitrito. Produce las enzimas $\alpha$ - y $\beta$-galactosidasa, $\mathrm{N}$-acetil $\beta$-glucosidasa, pirrolidonil arilamidasa (PYR), pirazinamidasa, siendo $\beta$-glucoronidasa, arginina dihidrolasa y $\alpha$-fucosidasa negativas. Presenta actividad sacarolítica sobre glucosa, maltosa, lactosa, sacarosa, xilosa, trehalosa, melibiosa y melezitosa, es arabinosa, manitol y ribosa variable y no utiliza glicógeno. En los últimos años han mejorado los métodos fenotípicos (galerías comerciales) y moleculares de identificación del género Actinomyces

Actinomyces spp habita las mucosas orofaríngea, genital e intestinal del hombre y los animales. Hasta la fecha se han descrito en la literatura científica cuatro aislados de A. urogenitalis, de orina, muestra uretral y vaginal asociada a uso de dispositivo intrauterino (DIU) de larga duración. El diagnóstico diferencial es con Actinomyces turicensis, la especie de mayor prevalencia en muestras de orina o tracto genital (Tabla 1). Actinomyces urogenitalis es sensible a penicilina, clindamicina, eritromicina, resistente a ciprofloxacina y con susceptibilidad variable a tetraciclina.

\begin{tabular}{|l|c|c|c|c|}
\hline \multicolumn{5}{|c|}{ Tabla 1. Esquema de diferenciación de Actinomyces urogenitalis y Actinomyces turicensis } \\
\hline Especie & \multicolumn{4}{|c|}{ Propiedad bioquímica } \\
\hline & Esculina hidrólisis & Nit2rato reducción & Lactosa fermentación & $\beta$-galactosidasa \\
\hline A. urogenitalis & + & + & + & + \\
\hline A. turicensis & - & - & - & - \\
\hline
\end{tabular}

\section{Referencias}

1.- Nikolaitschouk N, Hoyles L, Falsen E, Grainger JM, Collins MD. Characterization of Actinomyces isolates from samples from the human urogenital tract: description of Actinomyces urogenitalis sp. nov. Int J Syst Evol Microbiol 2000; 50: 1649-54.

\author{
María A. Martínez ${ }^{1}$, Alfredo Ovalle ${ }^{2}$ \\ ${ }^{1}$ Programa de Microbiología y Micología Facultad de Medicina Universidad de Chile \\ mamartin@med.uchile.cl \\ 2 Servicio y Departamento de Obstetricia, Ginecología y Neonatología, \\ Hospital San Borja Arriarán, Facultad de Medicina Universidad de Chile
}

